\title{
Timing Measurements from a TOF-PET Scanner Using Local PMT Triggering
}

\author{
Christopher C. M. Kyba, Rony I. Wiener, F. M. Newcomer, Member, IEEE, Richard Van Berg, Member, IEEE, \\ Nandor Dressnandt, and Joel S. Karp, Senior Member, IEEE
}

\begin{abstract}
The development of high light output scintillation crystals with fast decay times has made clinical whole body TOF-PET scanners possible. Current clinical systems based on LYSO have timing resolutions near 600ps (FWHM), but considerably better resolution is possible with faster scintillators. Bench top measurements have indicated that the LaBr3 modules used in a proto-type TOF PET scanner should allow for a resolution of 315-330ps. To achieve the best system timing resolution a new "local" triggering scheme has been developed, in which the seven PMTs used for energy and positioning determination are also used to produce a trigger. The electronics use fast components, and have been found to introduce a timing jitter of less than 50ps. The local triggering design reduces the effects of pulse pileup and dark current noise, greatly reducing the degradation of the timing resolution at higher rates. With these new electronics we have achieved a system time resolution of $420 \mathrm{ps}$ with little degradation at the rates expected for clinical FDG studies. The combination of the continuous design of our scanner and the local triggering scheme makes this design concept scalable to any configuration of PMTs in a full PET scanner.
\end{abstract}

\section{INTRODUCTION}

$\mathrm{T}$ HE development of high light output scintillation crystals with fast decay times has made clinical whole body TOF-PET scanners possible. For the commercial Gemini TF PET/CT scanner (Philips Medical Systems), it has been demonstrated that TOF reconstruction leads to improved image quality of whole body oncology studies, and in particular to improved lesion detectability and uptake estimation $[1,2]$. This system uses LYSO scintillators and has a system timing resolution of 600ps FWHM at low rates, and between 680-750ps FWHM resolution at typical clinical rates. Our group has previously reported preliminary results

Manuscript received November 23, 2007. This work was supported in part by NIH R33-EB001684 and R01-CA113941 and sponsored research agreements with Philips Medical Systems and Saint Gobain Crystals.

C. C. M. Kyba is with the Radiology Department, University of Pennsylvania, Philadelphia, PA 19143 USA (telephone: 215-349-5738, email:kyba@mail.med.upenn.edu).

R. I. Wiener, is with the Physics Department, University of Pennsylvania, Philadelphia, PA 19143 USA (telephone: 215-349-5738, e-mail: riwiener@hep.upenn.edu).

F. M. Newcomer is with the Physics Department, University of Pennsylvania, Philadelphia, PA 19143 USA (telephone: 215-898-4456, email: mitch@hep.upenn.edu).

R. Van Berg, is with the Physics Department, University of Pennsylvania, Philadelphia, PA 19143 USA (telephone: 215-898-5977, e-mail: rick@hep.upenn.edu).

N. Dressnandt, is with the Physics Department, University of Pennsylvania, Philadelphia, PA 19143 USA (telephone: 215-573-3380, email: nandor@hep.upenn.edu).

J. S. Karp is with the Radiology Department, University of Pennsylvania, Philadelphia, PA 19143 USA (telephone: 215-662-3073, e-mail: joelkarp@mail.med.upenn.edu). from a prototype whole body scanner ("LaPET") based on $\mathrm{LaBr}_{3}(5 \% \mathrm{Ce})$, in which we achieved a FWHM energy resolution of $7.5 \%$ and timing resolution of $460-500 \mathrm{ps}$ at low rates [3]. The limiting factor for improving the time resolution was found to be the triggering electronics, which had been adapted from commercial electronics. Based on the intrinsic properties of $\mathrm{LaBr}_{3}$ and previous bench top studies of the $\mathrm{LaBr}_{3}(5 \% \mathrm{Ce})$ detectors used in our scanner, we believe that a system time resolution of 315-330ps is attainable.

This paper presents a novel triggering technique that our group developed in order to improve the timing capabilities of the LaPET scanner. We present timing measurements obtained using this "local" triggering scheme, in which only the seven PMTs nearest to the hit crystal are used to produce the timestamp. The goal of the design was to provide the best possible timing resolution, and to minimize degradation of the timing resolution with increasing activity in the scanner.

\section{II. "LAPET" $\mathrm{LABR}_{3}$ SCANNER}

Our scanner is based on a pixelated Anger-logic detector, with a continuous light-guide coupled to individual pixels (see Fig. 1). The light-guide is used to spread the light from a crystal to allow position determination; the thickness chosen to result in light from individual crystals being shared between the seven PMTs nearest the crystal that absorbs the $511 \mathrm{keV}$ photon. Depending on the position of the crystal relative to the central PMT of the 7-PMT cluster, the set of seven PMTs collects between 80 and $93 \%$ of the total light emitted. The use of a hexagonal array increases photocathode coverage and minimizes losses in the gaps between the PMTs. The relative amount of light detected in each of the seven PMTs allows for crystal position determination, and the gamma energy may be determined by summing the light from the set of seven. The triggering, which will be described in detail later, is also based upon the sum of the signals of the seven PMTs. The PMTs are arranged to overlap separate blocks of $\mathrm{LaBr}_{3}$ crystals (detector modules), as is shown in the right hand panel of Fig 1 below. The full scanner is made up of a contiguous ring of 24 modules.
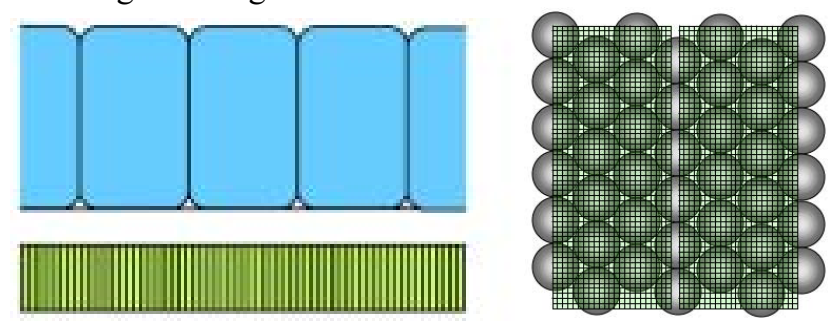
Fig. 1. Schematic of $\operatorname{LaBr}_{3}(5 \% \mathrm{Ce})$ Anger-logic detector (side and top views) with discrete crystals and continuous light-guide. In the left panel, the the PMTs (blue) are shown optically coupled to the crystals through a continuous light-guide. The image is not to scale. The right hand panel shows how detector modules are positioned relative to the PMTs. Each third column of PMTs straddles the gap between two $\mathrm{LaBr}_{3}$ modules. The gap between modules is narrower than the width of two crystals.

$\mathrm{LaBr}_{3}(5 \% \mathrm{Ce})$ was chosen as the scintillation crystal both for its fast decay time $(\mathrm{t} \sim 27 \mathrm{~ns})$ and for its very high light output $(60,000$ photons $/ \mathrm{MeV})$. This combination of properties results in very good energy resolution; $4 \%$ (FWHM) at 511 $\mathrm{keV}$ and coincidence timing resolution of $280 \mathrm{ps}$ (FWHM) [4]. In bench top measurements our group has found that coincidence time resolutions as good as 160ps can be achieved with small $\mathrm{LaBr}_{3}$ crystals coupled directly to very fast 1 -inch diameter Hamamatsu H4998 PMTs. In a practical PET detector, however, sensitivity and spatial resolution concerns require the use of long, narrow crystals. In bench top tests of small arrays of $4 \times 4 \times 30 \mathrm{~mm}^{3}$ crystals with arrays of 2-inch diameter Photonis XP20D0 PMTs, we observe coincidence time resolutions of $295 \mathrm{ps}$.

The scanner is made up of $24 \mathrm{LaBr}_{3}(5 \% \mathrm{Ce})$ modules coupled to a total of 432 Photonis XP20D0 PMTs. Each detector module built by Saint Gobain Crystals (Newbury, $\mathrm{OH})$ consists of $16204 \mathrm{~mm} \times 4 \mathrm{~mm} \times 30 \mathrm{~mm}$ crystals packaged in a 27 by 60 rectangular grid and isolated from each other with white reflective powder. Each module is imaged by 24 PMTs, 12 of which are coupled exclusively to that module and 12 of which are shared with the neighboring modules on each side. A flood of a portion of one module is shown in the right hand panel of Fig. 2. In bench top testing we observed an average energy resolution of 5.5\% (FWHM) and an average coincidence timing resolution between two arrays of $315 \mathrm{ps}$ (FWHM) [5], which is close to the resolution measured for the smaller arrays. The difference between the bench top and full scanner measurements reported in [3] was found to be due to the trigger scheme we had adapted from commercial electronics, leading us to move to a new triggering technique. These commercial electronics provided for only one trigger per module, and were designed for the LYSO based Gemini-TF. The timing jitter introduced by these electronics was not important for a system based on LYSO, but was the limiting factor for the more demanding timing measurements with the $\mathrm{LaBr}_{3}$ system.
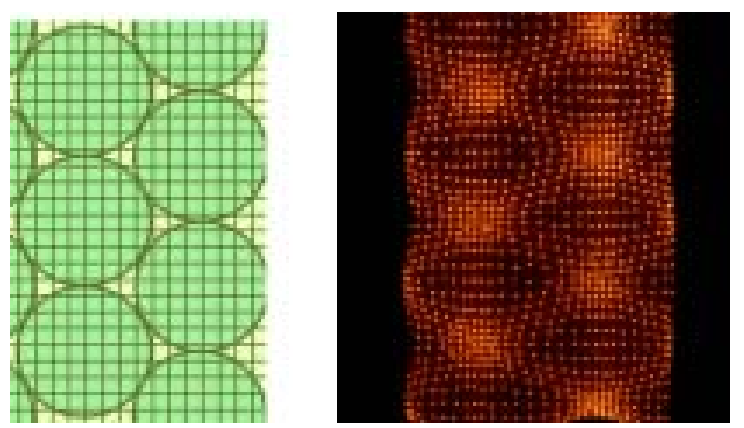

Fig. 2. An alternative top view of the detector is shown at left. On the right is a 2-D flood of a portion of one $\mathrm{LaBr}_{3}(5 \% \mathrm{Ce})$ detector module.

\section{LOCAL TRIGGER DESIGN}

The first issue to address in designing new triggering electronics is deciding upon the number of PMTs that should be used in each trigger zone. The earlier design, which suffered from significantly degraded time resolution with increasing rate, used 24 trigger zones, one for each $\mathrm{LaBr}_{3}$ module in the scanner. In each trigger zone, the signals from all of the PMTs in that zone were summed together, and the trigger time was defined by the time this analog sum signal crossed a programmable trigger threshold. While this configuration is guaranteed to collect all of the available light, in any given event most of the PMTs contribute only dark current background, as by design most of the light is incident on only the seven PMTs nearest the hit crystal. In addition, the larger the trigger zone, the larger the probability that an unrelated photon will affect the measurement at high count rates.

The "module sized" trigger zone is shown in the left panel of Fig. 3. The hit crystal is near the bottom left of the module, and is indicated by a white square. Regardless of which crystal in the module is hit, the signals from all of the PMTs are used to define the trigger. The second panel of Fig. 3 shows another possible trigger zone definition, in which the PMT with the highest charge is used to generate the trigger. In practice, this means that the PMT directly under the hit crystal will determine the trigger time. This design performs reasonably well when the crystal is close to the PMT center, in which case the PMT collects $\sim 65 \%$ of the available light. This design has problems, however, in the areas between the PMTs, where each of the nearest PMTs collects only $~ 35 \%$ of the available light. The timing resolution possible with this triggering arrangement varies from 350ps for crystals directly over a PMT center to $465 \mathrm{ps}$ for crystals halfway between two PMTs [6].
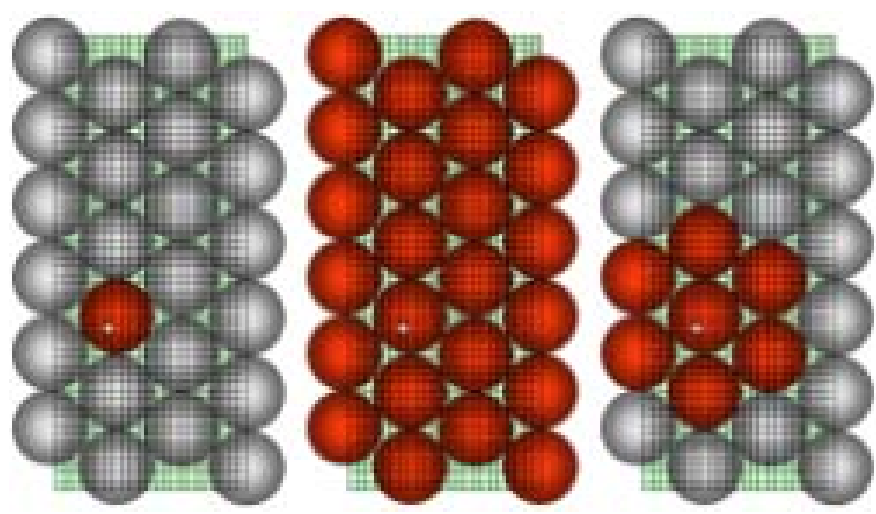

Fig. 3. The dark red PMTs show different options for defining trigger zones. Each circle represents a PMT, and the green grid represents a single $\mathrm{LaBr}_{3}$ module. The white square in each frame represents the hit crystal. At left is the large trigger zone definition used in the original triggering design. The center panel shows the conceptually simple technique of one trigger per PMT. The rightmost panel shows one zone in our local trigger design. The local trigger clusters maximize signal while minimizing inputs. Note that each PMT may be a part of several trigger zones; the center of the zone is under the hit crystal.

One example of a trigger zone definition used in our local triggering scheme is shown in the right panel of Fig. 3. The 
seven PMTs nearest the hit crystal collect most (80-93\%) of the light emitted, and each group of seven PMTs sees light from only a portion of the crystals in the module. For these reasons we expect the degradation in timing resolution due to pile up to be far smaller than for the large trigger zone definition on the left of Fig. 3. The seven PMT design is "local", in the sense that each PMT may contribute to several different sets of seven PMTs; the triggers overlap each other, and the most light will generally be collected by the set of seven closest to the hit crystal. The "gap" PMTs between modules are not used as local trigger centers, as light is not shared across the module boundaries.

A simplified block diagram of our custom trigger electronics is shown in Fig. 4. In order to make the local trigger possible each PMT center must be able to observe the signal in each of its neighbors. To accomplish this, each PMT signal is connected to seven emitter followers, making seven copies of the original signal. Each of the twelve trigger zones then use a current mirror to sum the signals from the central PMT and its six neighbors. This summed signal is passed to two leading edge discriminators. The "timing" discriminator employs common mode rejection, and its leading edge threshold is set very low in order to generate the timestamp based upon the earliest detected photons. Due to the low threshold, the timing discriminator fires nearly every time a nearby crystal is hit, regardless of the energy of the photon. Events with energies well below the photopeak are vetoed using the second "energy" discriminator. The signal from the timing discriminator is delayed relative to that from the energy discriminator, so that the timing of the output of the AND gate depends upon the timing of the "timing" discriminator.

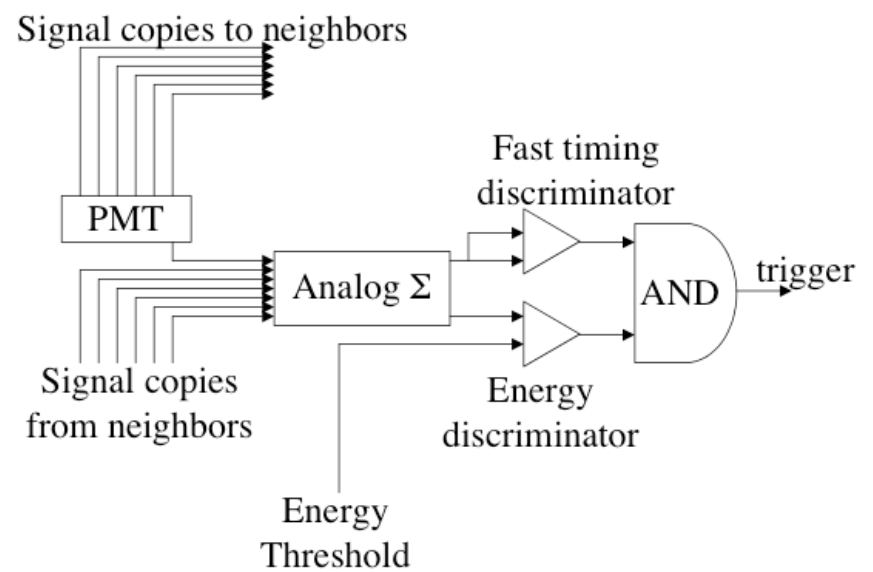

Fig. 4. Electronics Block diagram. Each trigger center receives signals from seven PMTs. Two leading edge discriminators are used, an "energy" discriminator is used reject events with energies below the photopeak, and the timing is determined by a discriminator with a low threshold. This allows our trigger to reject scatter events while maintaining a very low threshold for the timing measurement.

A generalized version of the timing discriminator circuit is shown in Fig. 5. The circuit is designed to eliminate the possibility of undesired low frequency oscillations introducing timing jitter. As can be seen in Fig. 5, the input signal is split into a "signal" (upper) and "threshold" (lower) paths.
The triggering threshold is set by changing the value of the uppermost resistor connecting the signal to $+\mathrm{V}$. At low frequencies the capacitor can be ignored, and the inductor acts as a wire. Thus, low frequency oscillations on the input to the circuit pass through to both inputs of the timing discriminator, achieving common mode rejection even in cases when the amplitude of the oscillation is larger than the triggering threshold. At high frequencies the capacitor acts as a wire, and the inductor acts as an attenuator. The input signal arrives unmodified at the upper discriminator input, while a greatly attenuated copy arrives at the lower (threshold) input.

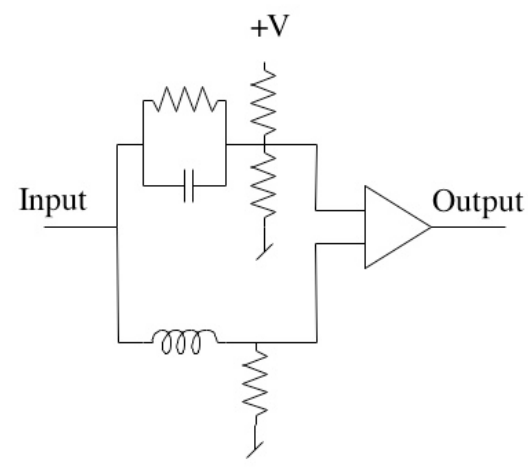

Fig 5. Schematic diagram for the threshold crossing timing discriminator with common mode rejection. If an undesired low frequency signal is input at the left of the schematic it will pass through to both inputs of the discriminator unperturbed. High frequency signals can pass through the top of the loop, but are attenuated by the inductor in the bottom part of the loop.

\section{Trigger Electronics PERFORMANCE}

The local triggering electronics have been integrated into the LaPET scanner, and are mounted in six positions on the front of the scanner, as is shown in Fig. 6. The intrinsic jitter introduced into the system by the electronics was measured using a pulser, and was found to be less than 50ps (FWHM). This is far smaller than the time resolution possible with the crystals and PMTs in the LaPET scanner, and is therefore effectively zero when added in quadrature to the fundamental limit imposed by the scintillator. The electronics do not contribute to any of the system resolution measurements reported below. 


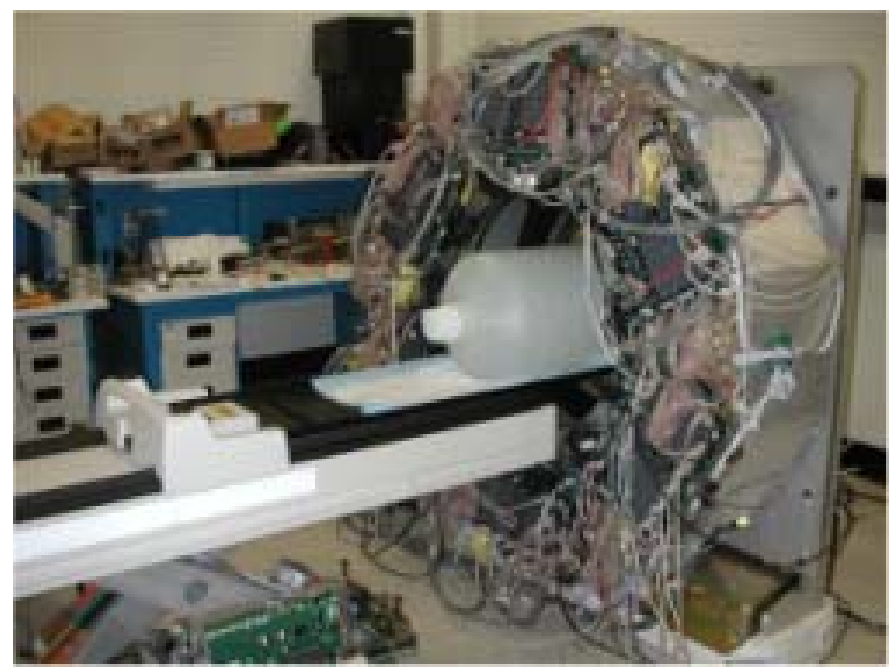

Fig. 6. The prototype scanner is shown with a large cylinder phantom extending outside the field of view. The new triggering electronics are positioned in six locations on the front of the scanner.

The individual crystal time offsets were calibrated using a rotating line phantom. In this measurement a capillary tube was filled with $250 \mu \mathrm{Ci}$ of ${ }^{18} \mathrm{~F}$ and mounted axially in the scanner at a radius of $20 \mathrm{~cm}$ from the center of the scanner. Coincident data were acquired while the tube was rotated, and in this way each crystal in the detector acquired events in coincidence with thousands of other crystals. We assume that the average time offset of the opposing crystals is zero, and an iterative algorithm was used to assign a unique offset to each crystal. This offset was then recorded in a lookup table, and was applied to data taken in list mode collection.

The absolute range of measured crystal timing offsets was found to be two nanoseconds. The tails of this distribution are due to malfunctioning electronics channels that have not yet been debugged. We have also used the rotating line data to measure the full system timing resolution, which we found to be 420ps FWHM. A histogram of the measured FWHM resolution for all of the crystals in the scanner is shown in Fig. 7. Again, the outliers are primarily due to a small number of malfunctioning channels.

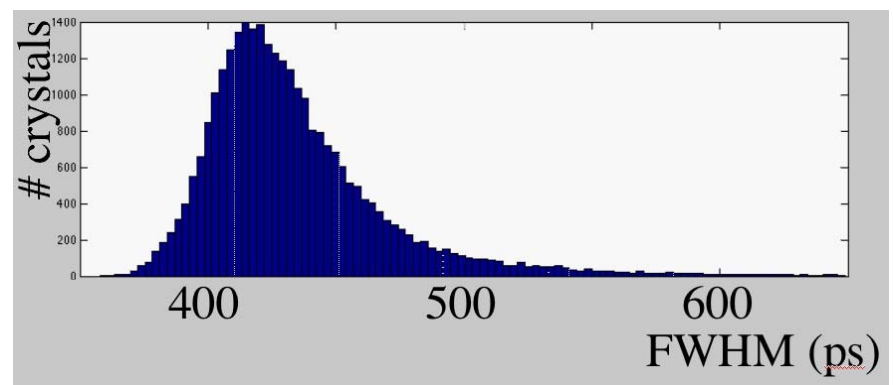

Fig. 7. Measured FWHM timing resolution for all of the crystals in the LaPET scanner. The system timing resolution was found to be $420 \mathrm{ps}$.

One of the primary goals of designing the local triggering electronics was to eliminate to the greatest degree possible the degradation of timing resolution with increasing singles rate. We have measured the system timing resolution as a function of rate using the setup shown in Fig. 8. $\mathrm{A}^{22} \mathrm{Na}$ point source (represented by the red dot in the figure) was placed in the center of the scanner, and the timing resolution of events reconstructing at that point were measured. Two uniform $20 \mathrm{~cm}$ diameter cylinders (the pink rectangles in the figure) were filled with ${ }^{18} \mathrm{~F}$ in order to elevate the singles rate. Measurements were taken over several hours as the activity in the cylinders decayed.

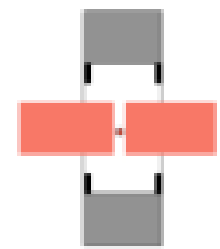

Fig. 8. Setup used to measure the timing resolution as a function of singles rate. $\mathrm{A}^{22} \mathrm{Na}$ point source was placed in the center of the scanner, and two uniform cylinder phantoms were used to elevate the singles rate.

The results of this measurement for the best pair of modules are shown in Fig. 9. The timing resolution is far more stable as a function of singles rate (or equivalently activity concentration) than it was with our original triggering electronics. At the rates expected for a clinical FDG study the timing resolution has degraded only slightly, and even at two to three times the expected clinical rate the timing resolution is still better than 500ps FWHM. The variation of the timing resolution at low rate for each pair of detector modules is shown in Fig. 10.

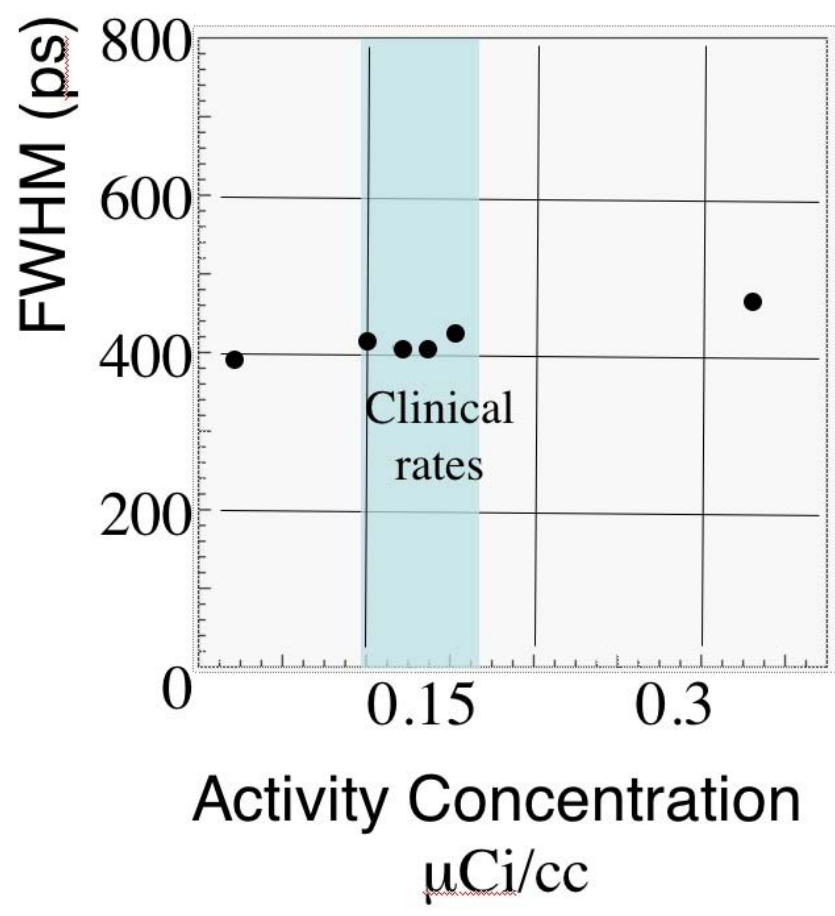

Fig. 9. Timing resolution is shown as a function of activity concentration (or equivalently singles rate) for one pair of detector modules. At the singles rate expected for a clinical FDG study only a slight worsening of resolution with increasing rate is observed. 


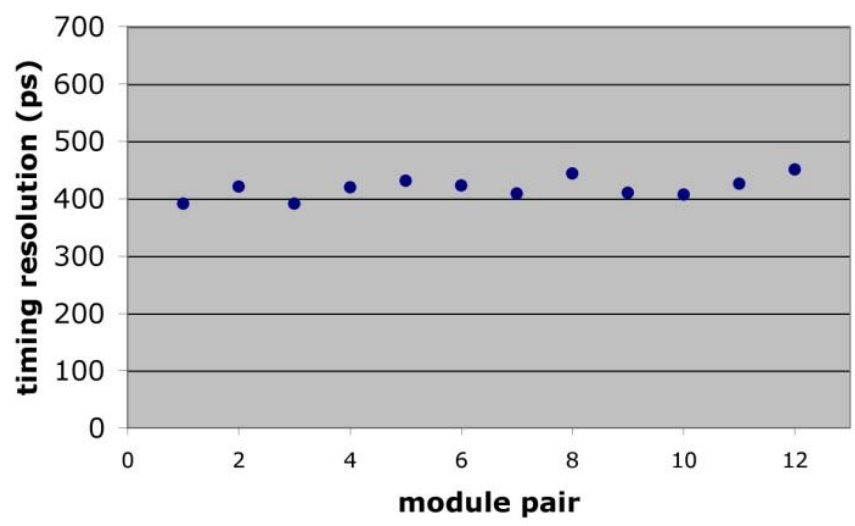

Fig 10. Timing resolution at low rate for each pair of detector modules. Each module is in coincidence with only the module directly opposite because the point source is centered.

\section{Phantom MEASUREMENTS}

The operation of the LaPET system was tested with phantom measurements. We used an iterative listmode reconstruction algorithm [3] to image several phantoms of different geometries and activity profiles. These measurements help us validate our calibrations for energy, crystal timing, and correction of random, scattered, and attenuated photons. While we intend to further optimize the scanner performance, these measurements serve as a benchmark for the performance of the scanner at $420 \mathrm{ps}$ resolution.

Our group has been performing clinical studies with the Gemini TF scanner since mid-2006. In these studies we have found that the incorporation of time-of-flight information into the reconstruction improves the detectability and quantification of small lesions, especially in the case of heavy patients. We have repeated on LaPET the lesion phantom experiment designed to simulate heavy patients that were described in [1]. In this experiment two cold and four hot spheres were placed in a $35 \mathrm{~cm}$ diameter cylinder phantom with a uniform background. The hot spheres were filled with an activity concentration of $6: 1$ with respect to the background. In order to simulate activity outside the field of view a $20 \mathrm{~cm}$ diameter by $30 \mathrm{~cm}$ long cylinder phantom was placed axially adjacent to the large diameter cylinder. The two smallest lesions had diameters of $10 \mathrm{~mm}$ and $13 \mathrm{~mm}$.

The results of this experiment are shown in Fig. 11. The data were reconstructed using both non-TOF (left) and TOF (right) reconstructions. The expected improvement in contrast due to the TOF reconstruction with 420ps timing resolution is easily visible The distortion at the bottom of the non-TOF image is caused by an imperfect model based attenuation and scatter correction. The transmission source attenuation correction is not yet operational, so the attenuation map was approximated and does not include the patient bed.

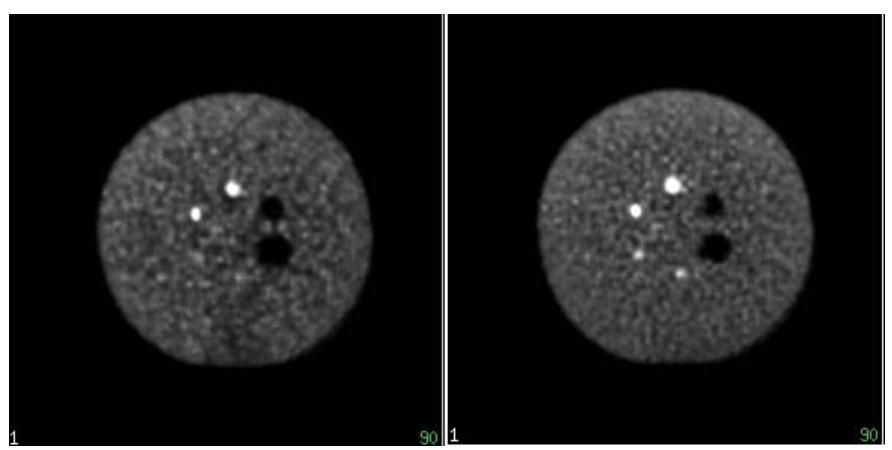

Fig 11. Lesion study on LaPET with a $35 \mathrm{~cm}$ diameter cylinder phantom. The same data were reconstructed with a non-TOF algorithm (left), and with a TOF algorithm with timing resolution of 420ps (right). The four hot spheres are at a 6:1 uptake activity relative to background, and all four spheres are clearly visible.

\section{CONCLUSIONS}

We have developed a novel "local" triggering scheme in which regardless of which crystal is hit, the group of seven PMTs nearest that crystal is used to produce the timestamp. In order to use the lowest possible triggering threshold we have employed a leading edge discriminator with common mode rejection. A second threshold crossing discriminator is used to reject events with energies under the photopeak.

Due to the scanner architecture, seven PMTs collect most of the light produced by each crystal; the inclusion of more than seven PMTs in the trigger would add little signal, but those extra PMTs would contribute dark current noise. In addition, the use of small trigger zones reduces the effects of pulse pileup at high rates. In our earlier measurements with the original electronics ( 1 trigger per detector module) this had resulted in degradation of the timing resolution with increasing activity in the scanner. While the triggering electronics were developed for the LaPET $\mathrm{LaBr}_{3}$ scanner, our "local" design could easily be adapted for a scanner with a different geometry and number of PMTs.

The performance of the triggering electronics was tested using the LaPET scanner. Individual crystal timing offsets were calibrated using a rotating line source, and these data were used to measure a system timing resolution of $420 \mathrm{ps}$ (FWHM). The module pair with the best resolution (390ps at low rate) was used to study the time resolution behavior as a function of rate. The electronics were found to have only a slight performance degradation at clinical rates, broadening to 460ps resolution at twice the approximate activity expected for a clinical FDG scan.

We have conducted preliminary phantom studies as an evaluation of system performance. These studies are very encouraging, as $10 \mathrm{~mm}$ and $13 \mathrm{~mm}$ spheres with a $6: 1$ uptake ratio are clearly visible in the TOF reconstructed images from a $35 \mathrm{~cm}$ diameter phantom. These results indicate that the $\mathrm{LaBr}_{3}$ scanner would achieve very good image quality in a challenging situation with a large patient due its excellent timing resolution.

The system timing resolution of $420 \mathrm{ps}$ observed on LaPET is improved compared to that reported in [3], and the significant degradation of timing resolution with increasing 
count rate observed in the earlier measurements has been greatly reduced. We have identified a number of areas in which we are able to further optimize the system. A small number of the electronics channels are known to have problems that affect their timing offset and timing resolution, and these channels will be repaired. We will optimize the leading edge threshold, as it is currently set very conservatively at about $1 / 10$ of the photopeak signal size. We intend to adjust the gains and transit time offsets of the PMTs through high voltage control, as currently the PMT gains differ by up to a factor of four, and the transit time offsets differ by roughly $1.5 \mathrm{~ns}$. We expect that the observed small degradation of timing resolution with increasing rate can be mitigated by applying pulse shaping (tail cancellation) to the PMT signals. Bench top measurements have indicated that the crystals and PMTs in the LaPET scanner should be able to perform at a timing resolution 315-330ps FWHM, and we believe that by performing these optimizations we will improve the system resolution to this level.

\section{ACKNOWLEDGMENT}

We thank Tom Heal, Austin Kuhn, Randall Kulp, Steve Martin, Godwin Mayers, Tyler Morgan-Wall, Gerd Muehllehner, Suleman Surti, and Matt Werner for their technical contributions.

\section{REFERENCES}

[1] Surti S, Kuhn A, Werner ME, Perkins AE, Kolthammer J, and Karp JS. Performance of Philips Gemini TF PET/CT Scanner with Special Consideration for Its Time-of-Plight Imaging Capabilities. JNM 48: 471-479, 2007.

[2] Karp JS, Surti, S, Daube-Witherspoon, ME, and Muehllehner G, The benefit of time-of-flight in PET imaging: Experimental and clinical results, JNM, 2008 in press

[3] Karp JS, Kuhn A, Perkins AE, Surti S, Werner ME, DaubeWitherspoon ME, Popescu LM, Vandenberghe S, and Muehllehner G, IEEE Nucl Sci Symp Conf Rec pp M04-8

[4] Shah KS, Glodo J, Klugerman M, Moses WW, Derenzo SE, Weber AJ. $\mathrm{LaBr} 3$ : Ce scintillators for gamma-ray spectroscopy. IEEE Trans Nucl Sci 50: 2410-2413, 2003.

[5] Kuhn A, Surti S, Karp JS, et al. Design of a Lanthanum Bromide Detector for TOF PET. IEEE Trans Nucl Sci 51: 2550-2557, 2004.

[6] Kuhn A, Surti S, Karp JS, Muehllehner G, Newcomer FM, VanBerg R. Performance assessment of pixelated $\mathrm{LaBr} 3$ detector modules for timeof-flight PET. IEEE Trans Nucl Sci 53: 1090 - 1095, 2006. 